\title{
SECURE COMMUNICATIONS OVER WIRELESS BROADCAST NETWORKS
}

\author{
Vikram Singh*1, Jaspal Ramola ${ }^{2}$ \\ ${ }^{* 1,2}$ (B.tech 3 rd sem ) dept. of computer science engineering, Dronacharya College of Engineering, Gurgaon, India \\ *1EmaI id: vikramsngh161@gmail.com
}

*Corresponding Author: -

Email ID: vikramsngh161@gmail.com

\begin{abstract}
: -
Wireless telecommunications is the transfer of information between two or more points that are not physically connected. Distances can be short, such as a few meters for television remote control, or as far as thousands or even millions of kilometers for deep-space radio communications. In this paper wireless broadcast network model (WBN) with secrecy constraints is investigated, in which a source node broadcasts confidential message flows to user nodes, with each message intended to be decoded accurately by one user and to be kept secret from all other users. In the existing system we developed, and implemented a compromised router detection protocol (DP) that dynamically infers, based on measured traffic rates and buffer sizes, the number of congestive packet losses (CPL) that will occur. Each and every packet is encrypted so that to prevent the data from eavesdropping. So the data is much secured.
\end{abstract}

Key words: -Application of wireless technology, types of wireless network, cellular network etc.

\section{(c) $(\$)$}




\section{INTRODUCTION}

Wireless operations permit services, such as long-range communications, that are impossible or impractical to implement with the use of wires. The term is commonly used in the telecommunications industry to refer to telecommunications systems (e.g., radio transmitters and receivers, remote controls, computer networks, network terminals, etc.) which use some form of energy (e.g., radio frequency (RF), acoustic energy, etc.) to transfer information without the use of wires.[1] Information is transferred in this manner over both short and long distances. Wireless networking (i.e., the various types of unlicensed $2.4 \mathrm{GHz}$ Wi-Fi devices) is used to meet many needs. Perhaps the most common use is to connect laptop users who travel from location to location.[1] Another common use is for mobile networks that connect via satellite. A wireless transmission method is a logical choice to network a LAN segment that must frequently change locations. The following situations justify the use of wireless technology:

$>$ To span a distance beyond the capabilities of typical cabling,

$>$ To provide a backup communications link in case of normal network failure,

$>$ To link portable or temporary workstations,

$>$ To overcome situations where normal cabling is difficult or financially impractical, or

$>$ To remotely connect mobile users or networks.[2]

\section{MODES}

Wireless communications can be via: -

$>$ Radio frequency communication

$>$ Microwave communication, for example long-range line-of-sight via highly directional antennas, or short-range communication,

$>$ Infrared (IR) short-range communication, for example from consumer IR devices such as remote controls or via Infrared Data Association (IrDA).

Applications may involve communication, point, broadcasting, cellular networks and other wireless networks.

\section{APPLICATIONS OF WIRELESS TECHNOLOGY}

Mobile Telephones

One of the best-known examples of wireless technology is the mobile phone, also known as a cellular phone, with more than 5.8 billion mobile cellular subscriptions worldwide as of the end of 2013. These wireless phones use radio waves to enable their users to make phone calls from many locations worldwide. They can be used within range of the mobile telephone site used to house the equipment required to transmit and receive the radio signals from these instruments.

\section{Wireless data communications}

Wireless data communications are an essential component of mobile computing. The various available technologies differ in local availability, coverage range and performance, and in some circumstances, users must be able to employ multiple connection types and switch between them. To simplify the experience for the user, connection manager software can be used, or a mobile VPN deployed to handle the multiple connections as a secure, single virtual network.

\section{Supporting technologies include: -}

Wi-Fi is a wireless local area network that enables portable computing devices to connect easily to the Internet. Standardized as IEEE 802.11 a,b,g,n, Wi-Fi approaches speeds of some types of wired Ethernet. Wi-Fi has become the de facto standard for access in private homes, within offices, and at public hotspots. Some businesses charge customers a monthly fee for service, while others have begun offering it for free in an effort to increase the sales of their goods.

Cellular data service offers coverage within a range of 10-15 miles from the nearest cell site. Speeds have increased as technologies have evolved, from earlier technologies such as GSM, CDMA and GPRS, to 3G networks such as WCDMA, EDGE or CDMA2000.

Mobile Satellite Communications may be used where other wireless connections are unavailable, such as in largely rural areas or remote locations. Satellite communications are especially important for transportation, aviation, maritime and military use.

\section{Wireless energy transfer}

Wireless energy transfer is a process whereby electrical energy is transmitted from a power source to an electrical load that does not have a built-in power source, without the use of interconnecting wires.

\section{Computer interface devices}

Answering the call of customers frustrated with cord clutter, many manufactures of computer peripherals turned to wireless technology to satisfy their consumer base. Originally these units used bulky, highly limited transceivers to mediate between a computer and a keyboard and mouse, however more recent generations have used small, high quality devices, some even incorporating Bluetooth. These systems have become so ubiquitous that some users have begun complaining about a lack of wired peripherals. Wireless devices tend to have a slightly slower response time than their wired counterparts, however the gap is decreasing. Concerns about the security of wireless keyboards arose at the end of 2007, when it was revealed that Microsoft's implementation of encryption in some of its $27 \mathrm{MHz}$ models was highly insecure. 


\section{TYPES OF WIRELESS NETWORKS}

\section{Wireless PAN}

Wireless personal area networks (WPANs) interconnect devices within a relatively small area, that is generally within a person's reach. For example, both Bluetooth radio and invisible infrared light provides a WPAN for interconnecting a headset to a laptop. ZigBee also supports WPAN applications. Wi-Fi PANs are becoming commonplace (2010) as equipment designers start to integrate Wi-Fi into a variety of consumer electronic devices. Intel "My WiFi" and Windows 7 "virtual Wi-Fi" capabilities have made Wi-Fi PANs simpler and easier to set up and configure.

\section{Wireless LAN}

A wireless local area network (WLAN) links two or more devices over a short distance using a wireless distribution method, usually providing a connection through an access point for Internet access. The use of spread-spectrum or OFDM technologies may allow users to move around within a local coverage area, and still remain connected to the network.

\section{Wireless mesh network}

A wireless mesh network is a wireless network made up of radio nodes organized in a mesh topology. Each node forwards messages on behalf of the other nodes. Mesh networks can "selfheal", automatically re-routing around a node that has lost power.

\section{Wireless MAN}

Wireless metropolitan area networks are a type of wireless network that connects several wireless LANs.

* WiMAX is a type of Wireless MAN and is described by the IEEE 802.16 standard.

\section{Wireless WAN}

Wireless wide area networks are wireless networks that typically cover large areas, such as between neighboring towns and cities, or city and suburb. These networks can be used to connect branch offices of business or as a public internet access system. The wireless connections between access points are usually point to point microwave links using parabolic dishes on the $2.4 \mathrm{GHz}$ band, rather than unidirectional antennas used with smaller networks. A typical system contains base station gateways, access points and wireless bridging relays. Other configurations are mesh systems where each access point acts as a relay also. When combined with renewable energy systems such as photo-voltaic solar panels or wind systems they can be standalone systems.

\section{Cellular network}

A cellular network or mobile network is a radio network distributed over land areas called cells, each served by at least one fixed-location transceiver, known as a cell site or base station. In a cellular network, each cell characteristically uses a different set of radio frequencies from all their immediate neighboring cells to avoid any interference.

When joined together these cells provide radio coverage over a wide geographic area. This enables a large number of portable transceivers (e.g., mobile phones, pagers, etc.) to communicate with each other and with fixed transceivers and telephones anywhere in the network, via base stations, even if some of the transceivers are moving through more than one cell during transmission.

Although originally intended for cell phones, with the development of smart phones, cellular telephone networks routinely carry data in addition to telephone conversations:

- Global System for Mobile Communications (GSM): The GSM network is divided into three major systems: the switching system, the base station system, and the operation and support system. The cell phone connects to the base system station which then connects to the operation and support station; it then connects to the switching station where the call is transferred to where it needs to go. GSM is the most common standard and is used for a majority of cell phones.

- Personal Communications Service (PCS): PCS is a radio band that can be used by mobile phones in North America and South Asia. Sprint happened to be the first service to set up a PCS.

- D-AMPS: Digital Advanced Mobile Phone Service, an upgraded version of AMPS, is being phased out due to advancement in technology. The newer GSM networks are replacing the older system.

\section{USES}

Some examples of usage include cellular phones which are part of everyday wireless networks, allowing easy personal communications. Another example, Inter-continental network systems, use radio satellites to communicate across the world. Emergency services such as the police utilize wireless networks to communicate effectively as well. Individuals and businesses use wireless networks to send and share data rapidly, whether it be in a small office building or across the world.

\section{General}

The industry accepts a handful of different wireless technologies. Each wireless technology is defined by a standard that describes unique functions at both the Physical and the Data Link layers of the OSI Model. These standards differ in their specified signaling methods, geographic ranges, and frequency usages, among other things. Such differences can make certain technologies better suited to home networks and others better suited to network larger organizations. 


\section{Performance}

Each standard varies in geographical range, thus making one standard more ideal than the next depending on what it is one is trying to accomplish with a wireless network. The performance of wireless networks satisfies a variety of applications such as voice and video. The use of this technology also gives room for future expansions. As wireless networking has become common place, sophistication increased through configuration of network hardware and software.

\section{Space}

Space is another characteristic of wireless networking. Wireless networks offer many advantages when it comes to difficult-to-wire areas trying to communicate such as across a street or river, a warehouse on the other side of the premise or buildings that are physically separated but operate as one. Wireless networks allow for users to designate a certain space which the network will be able to communicate with other devices through that network. Space is also created in homes as a result of eliminating clutters of wiring. This technology allows for an alternative to installing physical network mediums such as TPs, coaxes, or fiber-optics, which can also be expensive.

\section{Home}

For homeowners, wireless technology is an effective option as compared to Ethernet for sharing printers, scanners, and high-speed internet connections. WLANs help save from the cost of installation of cable mediums, save time from physical installation, and also creates mobility for devices connected to the network. Wireless networks are simple and require as few as one single wireless access point connected directly to the Internet via a router.

\section{Environmental concerns}

Starting around 2009, there have been increased concerns about the safety of wireless communications, despite little evidence of health risks so far. The president of Lake head University refused to agree to installation of a wireless network citing a California Public Utilities Commission study which said that the possible risk of tumors and other diseases due to exposure to electromagnetic fields (EMFs) needs to be further investigated.

Wireless access points are also often close to humans, but the drop off in power over distance is fast, following the inversesquare law. The HPA's position is that "...radio frequency (RF) exposures from Wi-Fi are likely to be lower than those from mobile phones.” It also saw "...no reason why schools and others should not use Wi-Fi equipment.” In October 2007, the HPA launched a new "systematic" study into the effects of Wi-Fi networks on behalf of the UK government, in order to calm fears that had appeared in the media in a recent period up to that time". Dr. Michael Clark, of the HPA, says published research on mobile phones and masts does not add up to an indictment of Wi-Fi.

\section{Conclusion}

The approach in this paper can be applied to analyze other wireless networks including Multiple- access, interference, and relay networks. This approach also allows the incorporation of public and common message flows for users in the system as well. In this paper we study modes of wireless communication, application of wireless network, cellular network and their uses and types of wireless network. Each and every packet is encrypted so that to prevent the data from eavesdropping. So, the data is much secured. Once the ambiguity from congestion is removed, subsequent packet losses can be attributed to malicious actions, still existing system is a good experiment but it will take more time while it's in the process. 